Original Article

\title{
CLINICAL SIGNIFICANCE OF POTENTIAL DRUG-DRUG INTERACTIONS AND THEIR TARGETS FOR MINIMIZATION AMONG HYPERTENSIVE DIABETIC OUTPATIENTS AT A KENYAN REFERRAL HOSPITAL
}

\author{
MAKITE SIMON LATI ${ }^{*}$, NYAMU GITONGA DAVID ${ }^{2}{ }^{*}$, ROSALINE NJOKI KINUTHIA ${ }^{2}$

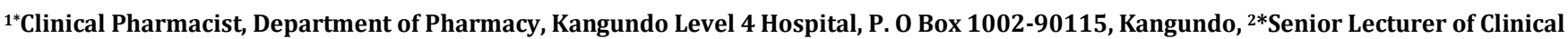 \\ Pharmacy, Department of Pharmaceutics and Pharmacy Practice, University of Nairobi, P. O. Box 19676-00202, Nairobi, ${ }^{2}$ Clinical \\ Pharmacist, Department of Pharmacy, Kenyatta National Hospital. P. O. Box 20723-00202 KNH, Nairobi \\ Email: makitesimonlati@gmail.com
}

Received: 21 Jun 2020, Revised and Accepted: 03 Aug 2020

\section{ABSTRACT}

Objective: To characterize the clinical significance of potential drug interactions and identify the targets for their minimization among adult diabetic hypertensive outpatients at Kenyatta National Hospital.

Methods: This cross-sectional study collected and analyzed data from 104 diabetic hypertensive outpatients (aged $\geq 18$ y) at the Department of Endocrinology Outpatient Clinic of Kenyatta National Hospital from 1st May 2019 to 31st August 2019. The main outcome measure was the clinical significance of potential drug interactions and the targets for minimization. Participants' sociodemographic data, drugs prescribed and targets for prevention of potential drug-drug interactions were extracted from patient medical records into predesigned data collection forms. Potential drug interactions were identified using the Micromedex® drug interaction checker. Data was exported to STATA® software version 13 for analysis.

Results: The study comprised predominantly females (70.2\%) and the mean age was $61.6( \pm 10.8)$ years. Over $80 \%$ of patients were receiving renin inhibitors or metformin and the commonest potential drug interaction $(25.0 \%)$ was antidiabetics-beta blockers. The most common potential clinical outcome of the drug-drug interaction was hyperkalemic lactic acidosis (14.4\%), induced by combining enalapril with metformin, and hypoglycemia (9.6\%) on concomitant use of antidiabetic and beta-blocker. Adverse clinical outcomes were mainly minimized through regular blood sugar checks (100\%), blood pressure monitoring (98.1\%), and minimal HbA1c (30.8\%) checks as well as serum urea and electrolytes (17.3\%) measurements.

Conclusion: There are potential adverse outcomes of combination pharmacologic therapies among diabetic hypertensive patients in Kenyatta National Hospital. Apart from the clinical monitoring, clinicians should be aware that diabetic hypertensive patients are likely to have serious adverse effects of drug interactions and, therefore, institute or intensify other measures such as arterial blood gases and serum electrolyte tests.

Keywords: Clinical outcomes, Potential drug-drug interactions, Antidiabetics, Antihypertensives, Diabetes, Hypertension, Kenya

(C) 2020 The Authors. Published by Innovare Academic Sciences Pvt Ltd. This is an open access article under the CC BY license (http://creativecommons.org/licenses/by/4.0/) DOI: http://dx.doi.org/10.22159/ijpps.2020v12i10.38816. Journal homepage: https://innovareacademics.in/journals/index.php/ijpps.

\section{INTRODUCTION}

Type 2 diabetes mellitus (DM) and hypertension are common comorbidities worldwide [1]. Furthermore, the prevalence of hypertension among DM patients is two to four-fold that of the normal population [2]. In 2015, the prevalence of DM was estimated at $9 \%$ globally (1) and $4 \%$ in Africa [3] while in Kenya approximately $12 \%$ of people are diabetic [4]. Studies have indicated that hypertensive patients usually exhibit insulin resistance and have a greater risk of developing DM than are normotensive individuals [5].

Diabetic hypertensive patients usually require a combination of drugs to achieve targeted blood glucose as well as blood pressure goals [6]. Studies have revealed that renin inhibitors such as Angiotensin Converting Enzyme inhibitors (ACEis) or Angiotensin Receptor Blockers (ARBs) are components of any drug regimen in the treatment of hypertension in diabetic patients [3]. In addition, depending on the level of blood pressure, incorporation of diuretics, $\beta$-blockers, and Calcium-Channel Blockers (CCBs) have been shown to improve the treatment outcome [7]. On the other hand, hypoglycaemic agents, including metformin, glibenclamide, insulin, thiazolinediones, and sitagliptins have proved to be effective in the management of diabetes among this patient population [8].

In an attempt to normalize the blood pressure and glucose levels, multiple drugs may predispose the patient to polypharmacy [9] and have the potential to interact, thereby producing undesirable effects [10]. Studies have also indicated that about $60 \%$ of patients may have drug interactions that will cause undesirable outcomes.
Several drug-drug interactions (DDIs) between antidiabetic drugs and some classes of antihypertensives have been documented, though many of these DDIs are of moderate clinical significance [11]. For instance, a study by Samardzic et al. found out that the most common DDI was attributed to thiazide diuretics (78.2\%) [12]. The potential DDI between metformin and hydrochlorothiazide was the most commonly reported (19.1\%) among DDIs related to a combination of thiazides and other antidiabetic or antihypertensive drugs [13].

Beta-blockers have also been implicated in increased hypoglycemic effect when used at the same time with sulfonylureas. Beta-blockers usually mask tachycardia as an initial symptom of hypoglycemia, with a higher incidence occurring with non-selective beta-blockers [14]. Beta-blockers such as bisoprolol or metoprolol are, therefore, safer for diabetic patients than nonselective beta-blockers [15]. There is, however, inadequate literature on the clinical outcomes of drug-drug interactions for diabetic hypertensive patients in resourceconstrained settings [4]. The present study characterized the clinical significance of potential drug interactions and the targets for their minimization among diabetic hypertensive adult outpatients at the largest teaching and referral hospital in Kenya.

\section{MATERIALS AND METHODS}

Study design and site

This was a cross-sectional study done among 104 adult patients between May to August 2019 at the largest teaching and referral 
hospital in East and Central Africa, Kenyatta National Hospital (KNH). The study was undertaken in a hospital clinic, which is the main entry point for patients diagnosed with DM. In addition, referrals of DM patients from all over the East and Central Africa are channeled through the clinic. Hospital records indicated that over $60 \%$ of patients who visit the study clinic are hypertensive or later develop secondary hypertension. Patients visiting the study clinic are usually enrolled for pharmacologic therapy and follow up.

\section{Study population}

Male and female, aged $\geq 18 \mathrm{y}$, were eligible if they had been diagnosed with comorbid HTN and DM, were on follow up at the clinic, and receiving at least one antihypertensive drug and one hypoglycemic agent. Due to ethical issues, special requirements in hypertension or diabetes management and challenges associated with direct patient interviews, pregnant women, patients with end-stage renal disease (ESRD) or liver disease, and patients with mental disorders were excluded.

\section{Sample size and sampling method}

The main outcome variable for this study was the outcome or clinical significance of potential DDIs among adult diabetic hypertensive patients. Previous studies on drug-drug interactions and their outcomes among patients with cardiovascular disease had indicated a prevalence of significant drug-drug interactions at 4-8 $\%[16,17]$. Using the formula for estimating the sample size for such epidemiological surveys [18] and the average prevalence rates from previous studies, the minimum sample size was 86 patients, which was embellished by $20 \%$ to make 104 participants to cater for data losses and non-responders. To achieve the required sample size during data collection, random sampling was used.

\section{Study methods}

Approval to conduct the study was granted by KNH/University of Nairobi-Ethics and Research Committee (KNH/UoN-ERC) vide reference KNH-ERC/A/192 and then registered by the Department of Research and Programs at KNH through number MED/42B/VOL.11/. Authority was also sought and granted from the Head of Department of Medicine at the hospital. Written and informed consent was sought from the eligible participants before the commencement of data collection.

A predesigned data collection form was used to capture participants' sociodemographic and other clinical characteristics, including the types of antidiabetics as well as antihypertensives prescribed. Potential drug-drug interactions between the drugs prescribed and clinical significance were assessed using Micromedex® drug interaction checker. Targets for minimization of drug interaction were also extracted from the medical files.

\section{Data entry and statistical analysis}

Raw data were entered into Microsoft Excel software version 2016 to create a database, cleaned, and exported to STATA $®$ statistical software version 13 for analysis. Summary statistics and frequencies of the sociodemographic and descriptive data on classes and types of drugs prescribed, interacting pairs of drugs and clinical outcomes of interactions as well as targets for minimization of the interactions were done.

\section{RESULTS}

Table 1 shows the sociodemographic characteristics of the respondents.

The female to male ratio was approximate 2.35: 1 and the mean age of the participants was $61.6( \pm 10.8)$ though the elderly patients comprised the majority (59.6\%). Majority of the participants had exceeded the ideal body weights (71.2 \%) and attended at least formal Kenyan education (table 1).

The pharmacological classes of the antidiabetics and antihypertensives prescribed are shown in fig. 1

Table 1: Sociodemographic characteristics of the study participants $(\mathrm{N}=104)$

\begin{tabular}{|c|c|c|c|}
\hline & & Participants & Percentage \\
\hline Variable & Characteristic & (n) & $(\%)$ \\
\hline \multicolumn{4}{|l|}{ Gender } \\
\hline & Male & 31 & 29.8 \\
\hline & Female & 73 & 70.2 \\
\hline \multicolumn{4}{|l|}{ Age (years) } \\
\hline & $18-45 y$ & 7 & 6.7 \\
\hline & $46-59 y$ & 35 & 33.7 \\
\hline & $>59 y$ & 62 & 59.6 \\
\hline \multicolumn{4}{|c|}{ Classes of Body weights/Body Mass Index } \\
\hline & Ideal (18.5-24.9) & 30 & 28.8 \\
\hline & Overweight (25.0-29.9) & 47 & 45.2 \\
\hline & Obese $(\geq 30.0)$ & 27 & 26.0 \\
\hline \multicolumn{4}{|l|}{ Marital status } \\
\hline & Single & 13 & 12.5 \\
\hline & Married & 68 & 65.4 \\
\hline & Separated & 1 & 1.0 \\
\hline & Widowed & 22 & 21.2 \\
\hline \multicolumn{4}{|l|}{ Religion } \\
\hline & Christians & 96 & 92.3 \\
\hline & Muslim & 8 & 7.7 \\
\hline \multicolumn{4}{|l|}{ Occupation } \\
\hline & Farmer & 15 & 14.4 \\
\hline \multirow{7}{*}{ Level of educationInformal } & Business/Self-Employment & 26 & 25.0 \\
\hline & Formal Employment & 16 & 15.4 \\
\hline & Unemployed/retired & 47 & 45.2 \\
\hline & & 5 & 4.8 \\
\hline & Primary & 35 & 33.7 \\
\hline & Secondary & 54 & 51.9 \\
\hline & College/University & 10 & 9.6 \\
\hline
\end{tabular}




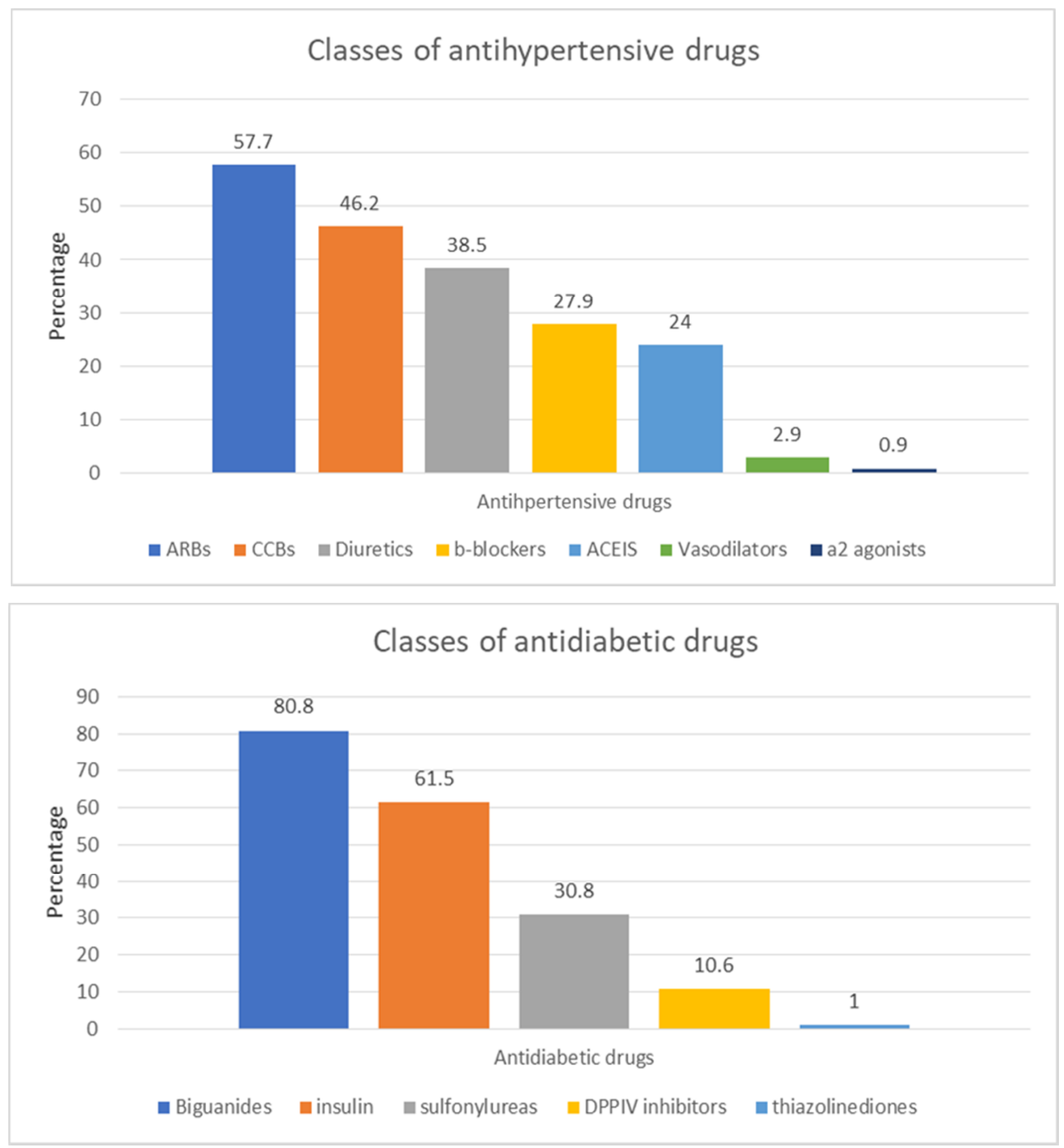

Fig. 1: Classes of antidiabetics and antihypertensives used by the Participants ( $\mathrm{N}=104)$, Key: $\mathrm{ACEI}=$ angiotensin-converting enzyme inhibitor; ARB=angiotensin receptor blocker $C C B=$ calcium channel blocker; DPPIV= dipeptidyl peptidase 4

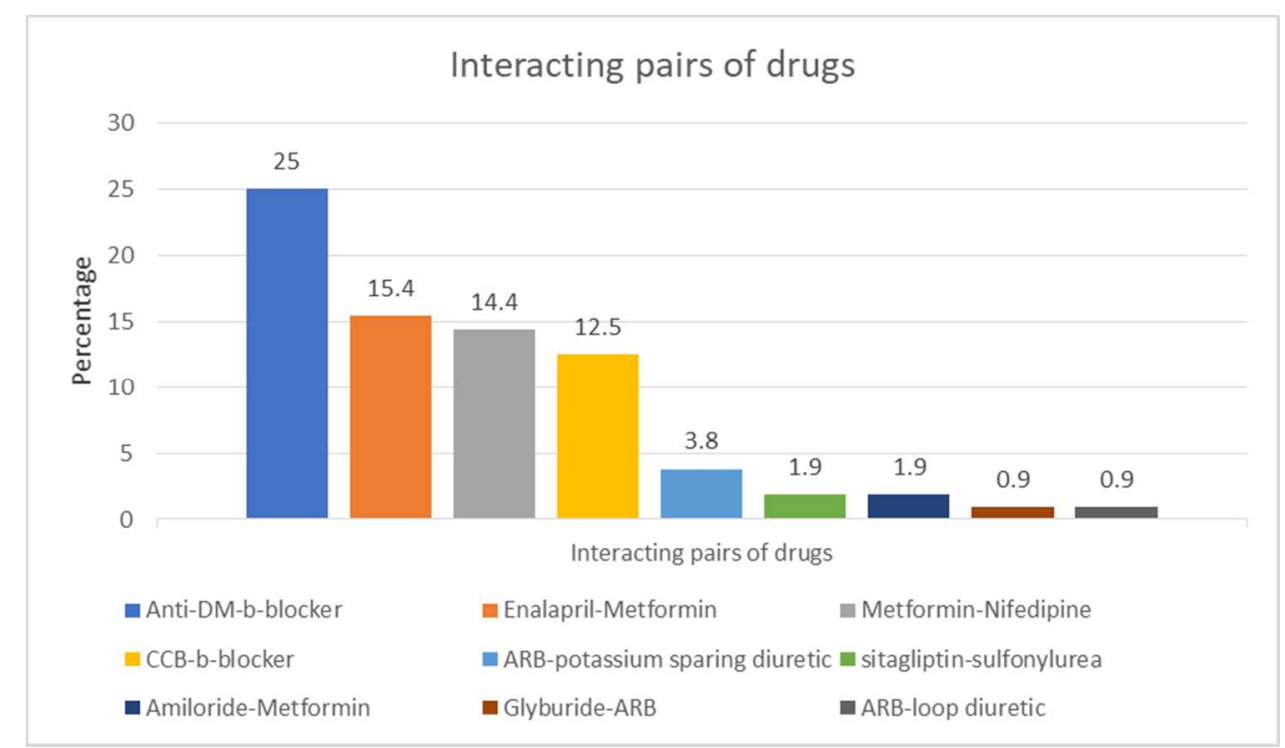

Fig. 2: Interacting pairs of antihypertensives and antidiabetics or both, Key-ARB=angiotensin receptor blocker; $\mathrm{CCB}=$ calcium channel blocker; DM=diabetes mellitus 
Biguanides were the most prescribed $(84,80.8 \%)$ antidiabetics, followed by insulin (61.5\%) and sulfonylureas (30.8\%). Antihypertensives prescribed principally were renin inhibitors at $81.7 \%$ followed by calcium channel blockers (46.2\%), diuretics $(38.5 \%)$ and $\beta$-blockers (27.9\%) in that order (fig. 1).

Fig. 2 shows the interacting drug combinations that were prescribed among the participants.
The most common drug combination that could result into a drugdrug interaction was antidiabetic drugs and a $\beta$-blocker $(26,25 \%)$ followed by enalapril and metformin $(16,15.4 \%)$, metformin and nifedipine $(15,14.4 \%)$ and, calcium channel blockers and $\beta$ blockers (12, 12.5\%). (fig. 2).

The clinical outcomes of potential drug-drug interactions are shown in fig. 3 below.

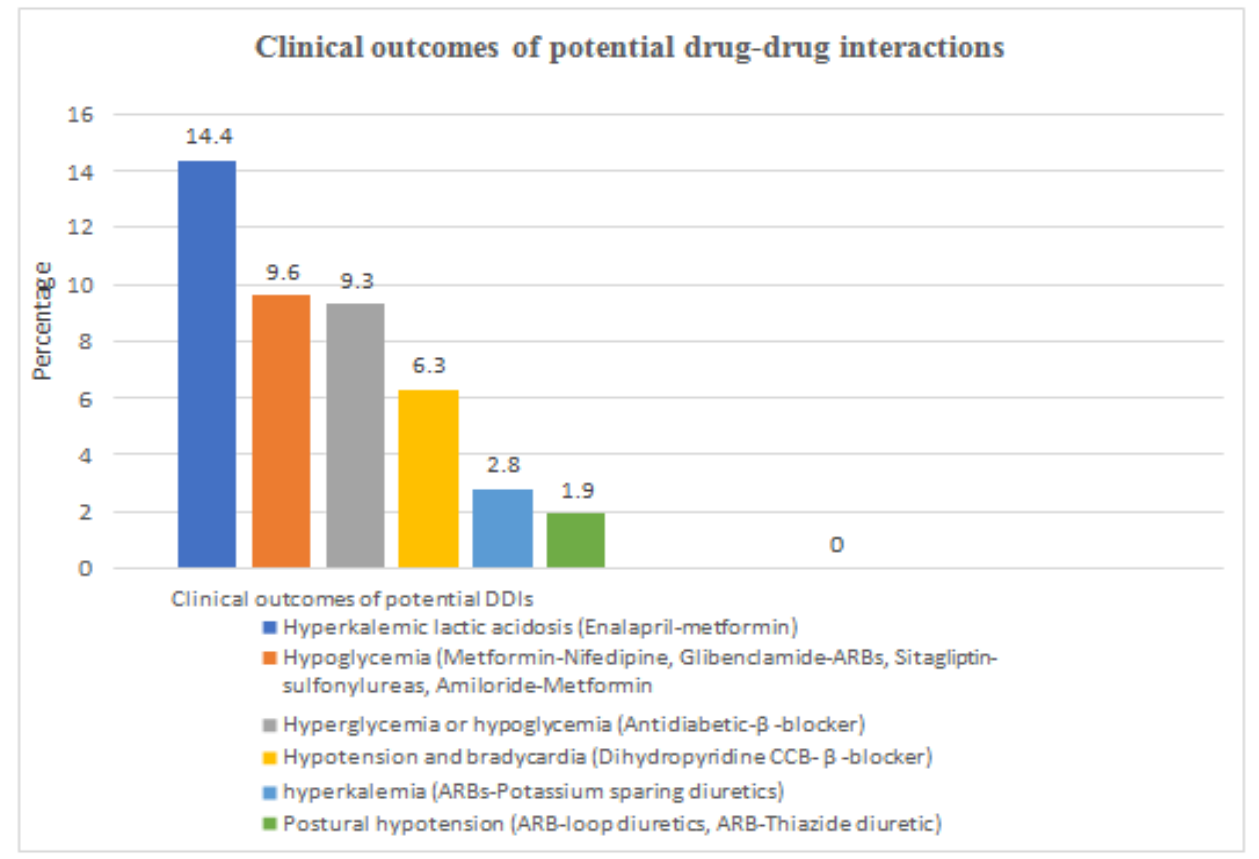

Fig. 3: Clinical outcomes of potential drug-drug interactions, Key: $\mathrm{ARB}=$ angiotensin receptor blocker, $\mathrm{CCB}=\mathrm{calcium}$ channel blocker, $\mathrm{DDI}=$ drug-drug interaction, $\mathrm{DM}=$ diabetes mellitus

The most common clinical outcome of the potential drug-drug interaction was hyperkalemic lactic acidosis $(14.4 \%)$ as a result of combining metformin-enalapril, followed by hypoglycemia (9.6\%) when metformin-nifedipine, glyburideARBs, amiloride-metformin, as well as the use of sitagliptinsulfonylureas, were concomitantly administered. Deranged glycemic control (9.3\%) from combining antidiabetics and B- blockers as well as bradycardia/hypotension was likely in patients on some non-dihydropyridine CCBs and B-blockers at $6.3 \%$ (fig. 3).

To identify the targets for the minimization of potential drug interactions, the frequency of monitoring parameters aimed at mitigating them are presented in fig. 4 .

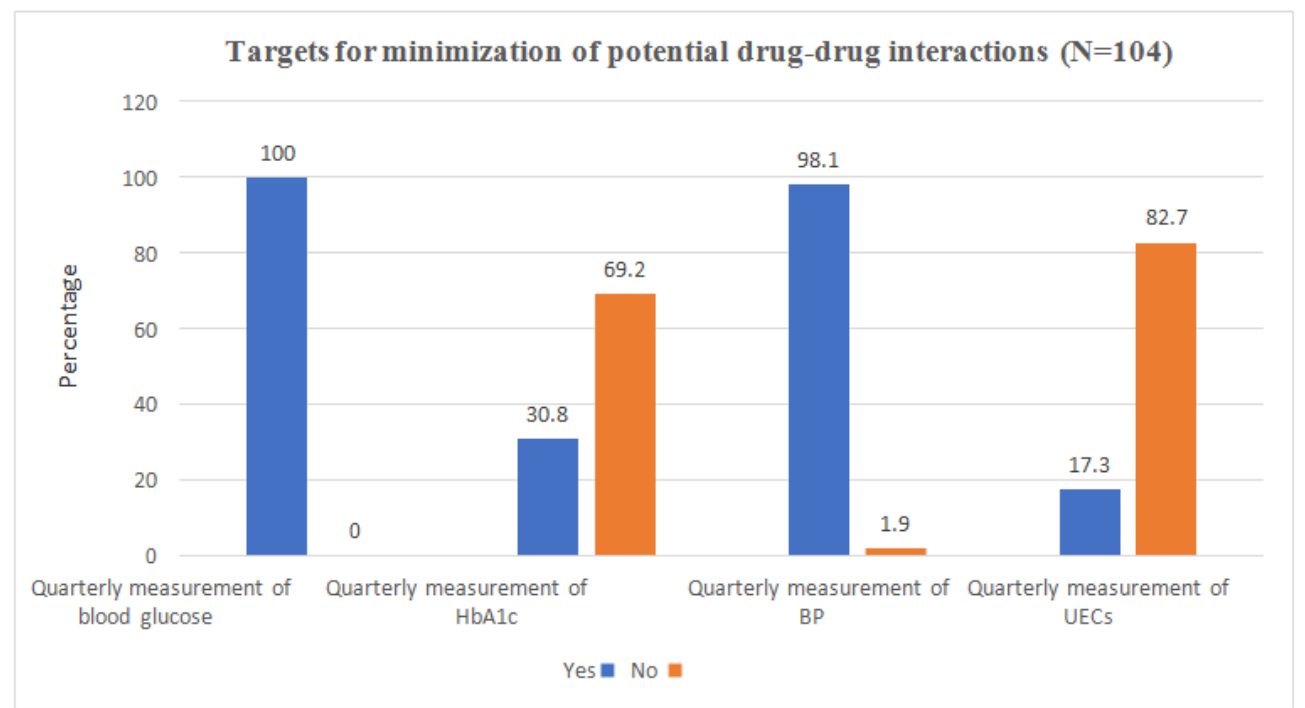

Fig. 4: Targets for minimization of potential drug-drug interactions, Key: Key-DDI= drug-drug interaction, HbA1C=hemoglobin A1C, UEC $=$ urea creatinine and electrolytes 
All study participants and $98.1 \%$ of them had blood sugar and blood pressure measured in the previous three weeks, respectively. Approximately a third (30.8\%) and $17.3 \%$ of the study population had their HbA1c and UECs levels determined in the previous three months prior to the study, respectively (fig. 4).

\section{DISCUSSION}

The present study documented the types of potential drug interactions among 104 adult patients with comorbid hypertension and diabetes in one of the largest teaching and referral hospitals in the East and Central Africa. Most of the patients were on the fifth decade of life as has been suggested in some studies that the prevalence of these comorbidities increases with advancing age [19].

Among the antidiabetics, metformin was the most prescribed hypoglycemic agent $(80.8 \%)$, while renin inhibitors were among the principle antihypertensives prescribed (81.7\%). Probably use of metformin was common because majority of the participants had exceeded the ideal body weights, and studies have suggested biguanides to be the drug of choice in obese patients [20]. There is also a possibility that clinicians were following the Kenya National Clinical Guidelines for Management of Diabetes Mellitus which recommends biguanides as first-choice drugs for the management of diabetes among the obese patients [21].

Research conducted by Bhatta et al. found metformin to be the most widely used antidiabetic drug at $40.5 \%$ in almost a similar population of patients [22]. The variation in the prevalence of metformin use, however, may be attributable to difference in patients' characteristics, study settings and the methods used. For example, unlike the present research, the latter study was prospective observational in nature which was done in India among diabetic patients only. Renin-inhibitors were widely prescribed probably due to their documented safety profile [23] as well as beneficial effects on the kidney and the heart among patients at risk of deterioration of these vital organs due to the comorbidities [24]. Other related studies have also revealed that majority of diabetic hypertensive patients also received at least one renin-inhibitors at $59 \%$ [25].

In the current study, the most common drug combination that could result into a drug-drug interaction was that of an antidiabetic drug and a $\beta$-blocker. Other mainly occurring interactions included enalapril and metformin, metformin and nifedipine and calcium channel blockers and $\beta$-blockers. The interacting pattern varies across studies conducted in different settings. For instance, Guantai et al. found that the most predominant interacting pair was enalapril and furosemide [17]. They also found potential drug-drug interactions between carvedilol and furosemide as well as insulin and furosemide. The differences in the pattern of drug-drug interactions may be due to differences in the study population. Furthermore, the study done by Guantai et al., unlike the current study, included hypertensive patients only, whereas the current study included patients with both diabetes and hypertension.

The most common potential clinical outcome of the drug-drug interaction was hyperkalemic lactic acidosis (14.4\%) as a result of combining metformin and enalapril. A case study reported by Weinberg et al. indicated that the use of metformin concurrently with ACE-Is significantly led to an increased risk of hyperkalemic lactic acidosis [26]. Metformin is known to cause lactic acidosis, especially when given to patients with poor renal function [27]. Enalapril, owing to the mode of action, may result into volume depletion and subsequently cause kidney injury, thereby precipitating lactic acidosis when used concomitantly with metformin. Studies have indicated that under such circumstances, metformin can accumulate to toxic levels and cause hyperkalemic lactic acidosis [28].

The Kenya National Clinical Guidelines for Management of Diabetes Mellitus recommends regular monitoring of blood sugar and blood pressure among diabetic hypertensive patients [21]. The revelations in the unanimous blood pressure and glucose measurements in the current study may be indicative of clinicians' awareness of the recommendations based on the huge proportion of patients monitored.

Whilst studies have indicated that HbA1c is the gold standard for monitoring and evaluation of long-term glycemic control in any diabetic patient on treatment [29], only a third of our patients had the levels checked in the previous three months. Related studies on glycemic control in patients with DM found out that quarterly monitoring of $\mathrm{HbA} 1_{\mathrm{C}}$ was also poorly done and only $39 \%$ of those on hypoglycemic agents performed the test [30]. The low frequency of monitoring of HbA1c levels in the present study may be due to the unavailability of the laboratory test or its prohibitive cost and hence the test may be reserved for the patients with erratic blood sugar controls. The low frequency of the determination of urea and electrolyte levels may also be attributable to the same reason.

\section{LIMITATIONS OF THE STUDY}

Although the study has significant findings for practice, we appreciate some limitations. Firstly, we only assessed the potential drug interactions among the antidiabetics and antihypertensives. We excluded other drugs that the patient may have been prescribed for other illnesses. In addition, the study was conducted over a short period of time and this may not be reflective of the occurrences throughout the year.

\section{CONCLUSION}

The potential drug-drug interactions were associated with unwanted clinical outcomes especially hyperkalemic lactic acidosis attributed to the use of metformin and enalapril. Apart from close monitoring blood sugars and blood pressures, clinicians should perform a patient-tailored investigation on $\mathrm{HbA} 1_{\mathrm{c}}$, urea and electrolytes and acid-base balance measurements among diabetic hypertensive patients on drug treatment. Future large studies are necessary to assess the impact of patients monitoring, the actual drug-drug interactions and the outcome of treatment.

\section{ACKNOWLEDGEMENT}

The authors wish to thank all staff and patients at KNH in the DEOC clinic for the immeasurable support during the study.

\section{FUNDING}

Nil

\section{AUTHORS' CONTRIBUTIONS}

SM and DN conceptualized the idea. SM wrote the concept, collected and analyzed the data. DN and RK assisted with proposal development, data analysis, and interpretation as well as drafting the manuscript. DN and RK reviewed the manuscript. All authors approved the final manuscript for publication.

\section{CONFLICT OF INTERESTS}

Authors declare no conflict of interest.

\section{REFERENCES}

1. Petrie JR, Guzik TJ, Touyz RM. Diabetes, hypertension and cardiovascular disease: clinical insights and vascular mechanisms. Can J Cardiol 2018;34:575-84.

2. Seedat Y, Rayner B. The abridged South African hypertension guideline 2011. South African Family Practice 2013;55:111-6.

3. Ogunsina MA, Anumah FO. Prevalence and correlates of hypertension and diabetes mellitus in an urban community in North-Western Nigeria. Pan Afr Med J 2018;8688:1-5.

4. Muchira J, Stuart Shor E, Kariuki J, Mukuna A, Ndigirigi I, Gakage L, et al. Distribution and characteristics of risk factors for cardiovascular-metabolic disease in a rural kenyan community. Int J Africa Nursing Sci 2015;3:76-81.

5. Mohan V, Seedat YK, Pradeepa R. The rising burden of diabetes and hypertension in Southeast Asian and African 
Regions: need for effective strategies for prevention and control in primary health care settings. Int J Hypertension 2013;1-14. DOI:10.1155/2013/409083

6. Shanmugam S. Chapter-28 management of hypertension in diabetes. Diabetes Mellitus; 2006. p. 124-8.

7. The health of the people: the African regional health report. Brazzaville: World Health Organization, Regional Office for Africa; 2006.

8. Manikandan R, Nandamuri SSS, Logaraj M. Assessment of medication adherence among patients with hypertension and diabetes mellitus in urban field practice. Asian J Pharm Clin Res 2018;11:130-2.

9. Maharani DD, Syafhan NF, Hersunaryati Y. Drug-related problems in hospitalized geriatric patients with diabetes mellitus. Int J Appl Pharm 2018;10:142-8.

10. Puspitasari AW, Azizahwati A, Hidayat AR. Analysis of potential drugs interaction on antihypertension drugs prescription in the community health center of sukmajaya district in period of june-november 2015. Asian J Pharm Clin Res 2017;10:61-5.

11. The safety of medicines in public health programmes: pharmacovigilance an essential tool. Geneva: World Health Organization; 2006.

12. Samardzic I. Incidence of potential drug-drug interactions with antidiabetic drugs. NCBI 2015;70:410-5.

13. Salvetti A. Thiazide diuretics in the treatment of hypertension: an update. J Am Soc Nephrol 2006;17 (4 Suppl 2):S25-9.

14. Sawicki PT, Siebenhofer A. Betablocker treatment in diabetes mellitus. J Int Med 2001;250:11-7.

15. Ostman J. Beta-adrenergic blockade and diabetes mellitus a review. Acta Med Scand Suppl 1983;672:69-77.

16. Ogamba E. Diabetes and hypertension in kisii teaching and referral. M Pharm Dissertation. University of Nairobi; 2016.

17. Guantai EM, Magot AA, Karimi PN, Maru SM, Nyamu DG. Identification and Characterization of potential drug interactions in hypertensive patients in a Kenyan Tertiary hospital Identification and characterization of potential drug interactions in Hypertensive patients in a Kenyan tertiary hospital. Afr J Pharmacol Ther 2018;7:7-12.

18. Cochran WG. Sampling techniques. $2^{\text {nd }}$ Ed. New York: John Wiley and Sons, Inc; 1963.
19. Kim MJ, Lim NK, Choi SJ, Park HY. Hypertension is an independent risk factor for type 2 diabetes: the korean genome and epidemiology study. Hypertension Res Nat Publishing Group 2015;38:783-9.

20. Lawrence JM, Reid J, Taylor GJ, Stirling C, Reckless JP. Favorable effects of pioglitazone and metformin compared with gliclazide on lipoprotein sub-fractions in overweight patients with early type 2 diabetes. Diabetes Care 2003;27:41-6.

21. Ministry of Health. National Clinical Guidelines for Management of Diabetes Mellitus-Republic of Kenya 2010;1:1-156.

22. Bhatta M. A prospective, cross-sectional study on cost and adherence of antidiabetic prescriptions at a tertiary care teaching hospital in South India. Value Health 2014;17:A746.

23. Abraham HMA, White CM, White WB. The comparative efficacy and safety of the angiotensin receptor blockers in the management of hypertension and other cardiovascular diseases. Drug Safety 2014;38:33-54.

24. Weir M. Effects of renin-angiotensin system inhibition endorgan protection: can we do better? Clin Ther 2007;29:1803-24.

25. Raval A, Dhanaraj E, Bhansali A, Yadav R. Prescription patterns of antihypertensive agents in T2DM patients visiting tertiary care centre in North India. Value Health 2013;16:1-9.

26. Weinberg JM. Risk of hyperkalemia in nondiabetic patients with chronic kidney disease receiving antihypertensive therapy. Arch Int Med 2009;169:1587-98.

27. Iftikhar H, Saleem M, Kaji A. Metformin-associated severe lactic acidosis in the setting of acute kidney injury. Cureus 2019;11:1-4.

28. Hsu WH, Hsiao PJ, Lin PC, Chen SC, Lee MY, Shin SJ. Effect of metformin on kidney function in patients with type 2 diabetes mellitus and moderate chronic kidney disease. Oncotarget 2018;9:5416-23.

29. Haghighatpanah M, Thunga G, Jha A, Mallayasamy S. Study on prescribing Pattern of anti-diabetic drugs among type 2 diabetes patients with a complication in South Indian teaching hospital. Asian J Pharm Clin Res 2016;9:194-7.

30. Harris MI. Frequency of blood glucose monitoring in relation to glycemic control in patients with type 2 diabetes. Diabetes Care 2001;24:979-82. 